\title{
EXTRAGALACTIC SOURCE COUNTS AT 24 MICRONS IN THE SPITZER FIRST LOOK SURVEY
}

\author{
Francine R. Marleau, ${ }^{1}$ D. Fadda, ${ }^{1}$ L. J. Storrie-Lombardi, ${ }^{1}$ G. Helou, ${ }^{1}$ D. Makovoz, ${ }^{1}$ D. T. Frayer, ${ }^{1}$ L. Yan, ${ }^{1}$ \\ P. N. Appleton, ${ }^{1}$ L. Armus, ${ }^{1}$ S. Chapman, ${ }^{2}$ P. I. Choi, ${ }^{1}$ F. Fang, ${ }^{1}$ I. Heinrichsen, ${ }^{1}$ M. Im, ${ }^{3}$ M. Lacy, ${ }^{1}$ D. Shupe, ${ }^{1}$ \\ B. T. Solfer, ${ }^{1,2}$ G. Seuires, ${ }^{1}$ J. Surace, ${ }^{1}$ H. I. Teplitz, ${ }^{1}$ and G. Wilson ${ }^{1}$ \\ Received 2004 March 29; accepted 2004 May 13
}

\begin{abstract}
We present the Spitzer Multiband Imaging Photometer $24 \mu \mathrm{m}$ source counts in the Extragalactic First Look Survey (FLS) main, verification, and European Large Area ISO Survey N1 fields. Spitzer's increased sensitivity and efficiency in large areal coverage over previous infrared telescopes, coupled with the enhanced sensitivity of the $24 \mu \mathrm{m}$ band to sources at intermediate redshift, dramatically improve the quality and statistics of number counts in the mid-infrared. The FLS observations cover areas of 4.4, 0.26, and $0.015 \mathrm{deg}^{2}$, respectively, and reach $3 \sigma$ depths of $0.11,0.08$, and $0.03 \mathrm{mJy}$. The extragalactic counts derived for each survey agree remarkably well. The counts can be fitted by a super-Euclidean power law of index $\alpha=-2.9$ from 0.2 to $0.9 \mathrm{mJy}$, with a flattening of the counts at fluxes fainter than $0.2 \mathrm{mJy}$. Comparison with infrared galaxy evolution models reveals a peak's displacement in the $24 \mu \mathrm{m}$ counts. This is probably due to the detection of a new population of galaxies with redshift between 1 and 2, previously unseen in the $15 \mu \mathrm{m}$ deep counts.
\end{abstract}

Subject headings: galaxies: statistics — infrared: galaxies

\section{INTRODUCTION}

The mid- and far-infrared regions of the electromagnetic spectrum probe the population of starburst galaxies obscured by dust. Counting galaxies as a function of mid- and farinfrared fluxes therefore puts new limits on the inferred geometry of the universe. Moreover, using counts at different wavelengths and extragalactic background light measurements, it is possible to constrain models of galaxy evolution (Franceschini et al. 2001; Xu et al. 2003; Lagache et al. 2003, hereafter LDP03; Chapman et al. 2003; Chary \& Elbaz 2001). Source counts studies have generally been done in the optical because of the advantage of good sensitivity and resolution of optical instruments, but optical counts are biased by dust obscuration, and the task of correcting for dust extinction is difficult (Calzetti 1997). The optical counts reveal a strong evolution of the population of blue galaxies as a function of redshift to $z=1$ (Metcalfe et al. 1995; Lilly et al. 1996). At longer near-infrared wavelengths, where the light observed samples the old stellar population, the galaxy counts show a passive luminosity evolution (Gardner et al. 1993; Yan et al. 1998).

Counts from mid- and far-infrared deep surveys also point to a strong evolution. IRAS unveiled a new population of luminous infrared galaxies (LIRGs; $L>10^{11} L_{\odot}$ ) that emit the bulk of their energy beyond $60 \mu \mathrm{m}$ (e.g., Soifer et al. 1987). The IRAS galaxy counts revealed an excess of faint sources compared to no-evolution models (Hacking et al. 1987). The deep ISOCAM survey at $15 \mu \mathrm{m}$ discovered a population of dust-obscured galaxies at $z=0.8$ with LIRG-like luminosities (Elbaz et al. 1999, 2002; Flores et al. 1999). The source counts derived from the deep surveys cover the flux density range 0.05-4 mJy, showing a significant super-Euclidean slope from

\footnotetext{
Spitzer Science Center, California Institute of Technology, Pasadena, CA 91125.

${ }^{2}$ California Institute of Technology, Pasadena, CA 91125

3 Astronomy Program, School of Earth and Environmental Sciences, Seoul National University, Shillim-dong, Kwanak-gu, Seoul, South Korea.
}

3 to $0.4 \mathrm{mJy}$ and a change of slope at flux densities fainter than $0.4 \mathrm{mJy}$. The European Large Area ISO Survey (ELAIS; Oliver et al. 2000) has provided source counts in the flux density range $0.45-150 \mathrm{mJy}$, linking the IRAS counts to the deep ISOCAM counts (Gruppioni et al. 2002).

The Spitzer Space Telescope (Werner et al. 2004) was launched on 2003 August 25, and its three cryogenically cooled science instruments combine to provide imaging and spectroscopy from 3 to $180 \mu \mathrm{m}$ with orders-of-magnitude improvements in capability over previous infrared telescopes. Compared to the $15 \mu \mathrm{m}$ band whose sensitivity is enhanced for sources in the redshift range $0.5-1.5$ as a result of the prominent polycyclic aromatic hydrocarbon (PAH) features, the Spitzer $24 \mu \mathrm{m}$ band makes it possible to explore the universe up to $z=2.5$. Coupled with better sensitivity and increased efficiency in large areal coverage, the $24 \mu \mathrm{m}$ observations with Spitzer dramatically improve the quality and statistics of number counts in the mid-infrared. The First Look Survey ${ }^{4}$ (FLS) was the first survey undertaken by Spitzer. The purpose of this survey is to characterize the mid-infrared sky at previously unexplored depths and includes an extragalactic, Galactic, and ecliptic component.

In this paper, we present source counts at $24 \mu \mathrm{m}$ in the Spitzer FLS main and verification surveys as well as in the ELAIS-N1 region, which has deeper observations. The surveys are described in $\S 2$. We present the data reduction and flux extraction method in $\S 3$. In $\S 4$, we describe the sample selection and the $24 \mu \mathrm{m}$ counts. Finally, in $\S 5$, we discuss our results and their implications.

\section{DESCRIPTION OF THE SURVEYS}

The extragalactic component of the Spitzer FLS covers a $4.4 \mathrm{deg}^{2}$ region near the ecliptic pole $\left(17^{\mathrm{h}} 18^{\mathrm{m}} 00^{\mathrm{s}},+59^{\circ} 30^{\prime} 00^{\prime \prime}\right.$, J2000.0) and was observed by Spitzer in 2003 December for a total of $62.6 \mathrm{hr}$ of Multiband Imaging Photometer for Spitzer (MIPS; Rieke et al. 2004) and Infrared Array Camera

\footnotetext{
${ }^{4}$ See http://ssc.spitzer.caltech.edu/fls.
} 
TABLE 1

Spitzer FLS $24 \mu \mathrm{m}$ SURVEYS

\begin{tabular}{ccrcc}
\hline \hline Name & $\begin{array}{c}\text { Area } \\
\left(\mathrm{deg}^{2}\right)\end{array}$ & \multicolumn{1}{c}{$\begin{array}{c}\left\langle t_{\text {int }}\right\rangle \\
(\mathrm{s})\end{array}$} & $\begin{array}{c}\text { Depth }(3 \sigma) \\
(\mathrm{mJy})\end{array}$ & $\begin{array}{c}\text { Completeness }(80 \%) \\
(\mathrm{mJy})\end{array}$ \\
\hline Main .............. & 4.388 & 84 & 0.11 & 0.23 \\
Verification ...... & 0.259 & 349 & 0.08 & 0.16 \\
ELAIS-N1 ...... & 0.015 & 4268 & 0.03 & 0.09 \\
\hline
\end{tabular}

(IRAC; Fazio et al. 2004) imaging. A $0.26 \mathrm{deg}^{2}$ verification survey within the main field $\left(17^{\mathrm{h}} 17^{\mathrm{m}} 00^{\mathrm{s}},+59^{\circ} 45^{\prime} 00^{\prime \prime}, \mathrm{J} 2000.0\right)$ with exposure times 4 times that of the main survey was taken to allow accurate characterization of the completeness and reliability of source detections in the main field. In addition, deeper observations were carried out in the ELAIS-N1 region $\left(16^{\mathrm{h}} 10^{\mathrm{m}} 01^{\mathrm{s}},+54^{\circ} 30^{\prime} 36^{\prime \prime}, \mathrm{J} 2000.0\right)$ as part of the Spitzer Director's Discretionary Time program to test the confusion limit at $24 \mu \mathrm{m}$ (L. J. Storrie-Lombardi et al. 2004, in preparation). The main characteristics of the three surveys are given in Table 1.

\section{DATA REDUCTION}

The FLS data were processed using version S8.9 of the Spitzer Science Center (SSC) pipeline (see Spitzer Observer Manual). ${ }^{5}$ Further corrections for illumination and flat-field variations, which depend on the scan mirror position, were derived from the data and applied to the individual frames (D. Fadda et al. 2004, in preparation). Saturated sources and bright cosmic rays can produce a flux drop every fourth column associated with the readouts. We identified the affected frames and applied an additive correction to the columns with low flux. The frames were co-added using the SSC software Mopex ${ }^{6}$ to obtain a mosaic with half the original pixel scale $(1$ "'27). The projection was done using a linear interpolation, taking into account the distortion corrections. The initial projection utilized bad pixel masks produced by the pipeline. Further pixels affected by cosmic rays were flagged using a multiframe temporal outlier detection, and the images were reprojected using these improved masks.

Given the resolution of MIPS at $24 \mu \mathrm{m}$ (the point-spread function [PSF] FWHM measures 5"9), typical extragalactic sources appear as point sources. For this reason, we can perform source extraction using a PSF fitting algorithm (StarFinder; Diolaiti et al. 2000). The StarFinder code has been developed to analyze adaptive optics images of very crowded stellar fields. We find that this extraction method performs remarkably well in the case of the $24 \mu \mathrm{m}$ images because of the good sampling of the PSF. Compared to the IRAF package DAOPHOT, this method performs a better evaluation of the PSF on the image because it evaluates the background emission over the entire frame and iteratively estimates the PSF after each extraction (see Aloisi et al. 2001). We compared the flux measurements obtained with StarFinder and SExtractor (Bertin \& Arnouts 1996) in the region observed in both the shallow and verification surveys. The scatter in the difference between the shallow and deep flux measurements of faint sources is larger for the SExtractor aperture-corrected fluxes (see Fig. 1). The aperture used for SExtractor is 3 times FWHM ( $\sim 70 \%$ of the PSF total

\footnotetext{
${ }^{5}$ See http://ssc.spitzer.caltech.edu/documents/som.

${ }^{6}$ See http://ssc.spitzer.caltech.edu/postbcd.
}

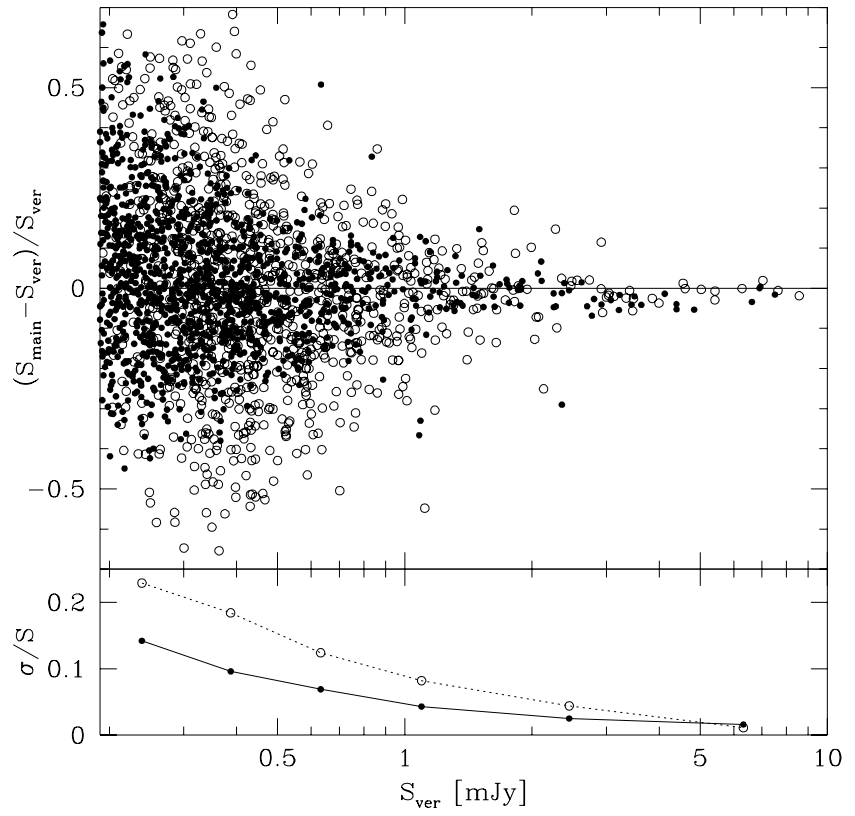

FIG. 1.-Comparison between the StarFinder ( filled circles) and SExtractor (open circles) fluxes measured in the shallow and deep verification region. The lower panel shows the photometric error as a function of flux for each source extraction method. The scatter in the difference between the shallow and deep flux measurements of faint sources is larger for the SExtractor aperture corrected fluxes.

flux). The relative error of the flux extracted by StarFinder is also shown in Figure 1. StarFinder permits a more reliable flux measurement because it recovers $90 \%$ of the flux directly and deblends sources more efficiently than SExtractor. In the case of deep fields, deblending is essential for obtaining reliable counts. Point source fluxes were corrected to total fluxes using MIPS $24 \mu \mathrm{m}$ calibrators. The main field contains several extended sources such as nearby galaxies and bright IRAS sources. We identified 79 extended sources by visual inspection and measured their fluxes inside suitable apertures after subtracting surrounding point sources.

The spacecraft astrometry (known to better than $1^{\prime \prime}$ ) was used to co-add the frames. A simple offset correction (on the order of $1^{\prime \prime}$ in right ascension) was applied to the final mosaic by matching sources to the Very Large Array ${ }^{7}$ counterparts (Condon et al. 2003).

\section{THE $24 \mu \mathrm{m}$ EXTRAGALACTIC SOURCE COUNTS}

For each field, we considered the part of the mosaic that showed the most uniform coverage (number of frames coadded). We also removed a $0.24 \mathrm{deg}^{2}$ area in the main field that contains cirrus emission, which can affect the flux measurement of sources. We counted sources in the remaining areas $\left(4.15,0.26\right.$, and $\left.0.015 \mathrm{deg}^{2}\right)$ with uniform coverage (median of 23, 93, and 139 frames) for the main, verification, and ELAIS-N1 surveys, respectively.

An essential step to produce reliable counts is to discriminate stars from extragalactic sources. The star contamination at $24 \mu \mathrm{m}$ is expected to be low because we are sampling the tail of the Rayleigh-Jeans energy distribution and observing at

\footnotetext{
${ }^{7}$ The VLA is operated by the National Radio Astronomy Observatory, which is a facility of the National Science Foundation, operated under cooperative agreement by Associated Universities, Inc.
} 


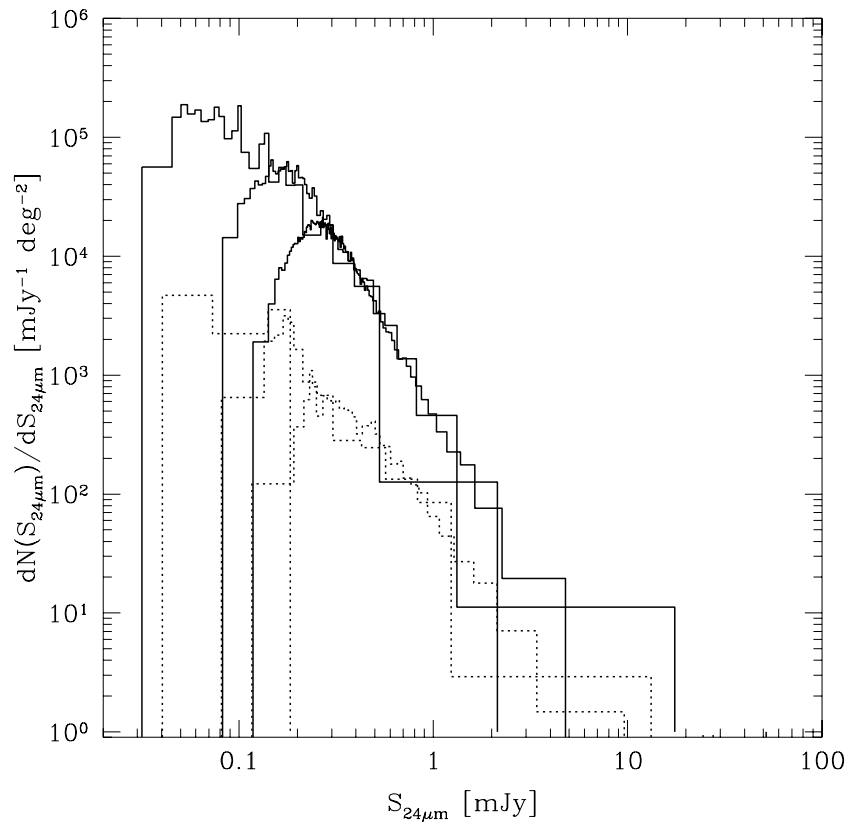

FIG. 2.-Differential extragalactic source counts at $24 \mu \mathrm{m}$ for the main (solid line, shallow counts), verification (solid line, middle counts) and ELAIS-N1 (solid line, deep counts) surveys. The contribution of stars is displayed as separate histograms for each survey (dotted line).

high Galactic latitude. Stars are identified on the basis of their optical counterparts. For stars brighter than $R=19$, we rely on the star classification of the Sloan Digital Sky Survey (D. W. Hogg et al. 2004, in preparation). At fainter magnitudes, we used the stellarity index measured with SExtractor on a deep $R$-band image (Fadda et al. 2004). This index is reliable to $R<23$, beyond which we expect a negligible contribution of stars at $24 \mu \mathrm{m}$. We find $8 \%$ stellar contribution for fluxes brighter than $0.3 \mathrm{mJy}$ in the main field, $3 \%$ in the flux range $0.2-0.3 \mathrm{mJy}$ in the verification survey, and $4 \%$ in the $0.1-0.2 \mathrm{mJy}$ range.

The completeness of the main survey was estimated using the verification region for which both shallow and deep observations were taken. Using the deeper verification counts, we find that the counts in the main survey are $100 \%$ complete to a flux of $0.28 \mathrm{mJy}(80 \%$ complete at $0.23 \mathrm{mJy})$. Similarly, using the deep ELAIS-N1 counts, we were able to measure a completeness limit of $0.21 \mathrm{mJy}(80 \%$ complete at $0.16 \mathrm{mJy})$ for the verification survey. Simulations for completeness and reliability measurements for the ELAIS-N1 field can be found in L. J. Storrie-Lombardi et al. (2004, in preparation). The completeness limits of each survey are listed in Table 1 . The $3 \sigma$ limiting depth, also given in Table 1, was computed by measuring the noise (standard deviation) in the mosaic. To compute the total limiting flux, we assumed a Gaussian PSF with 5".9 FWHM normalized to the flux measured in the central pixel.

\section{DISCUSSION AND CONCLUSIONS}

The extragalactic (solid line) and stellar counts (dotted line) are shown in Figure 2. The counts from the three surveys agree remarkably well, as shown by the KolmogorovSmirnov test. The probability that the two flux distributions (ELAIS-N1 vs. verification and verification vs. main) are not the same is less than $20 \%$ and $15 \%$, respectively. The counts flatten at fluxes fainter than $0.2 \mathrm{mJy}$. For fluxes between 0.1

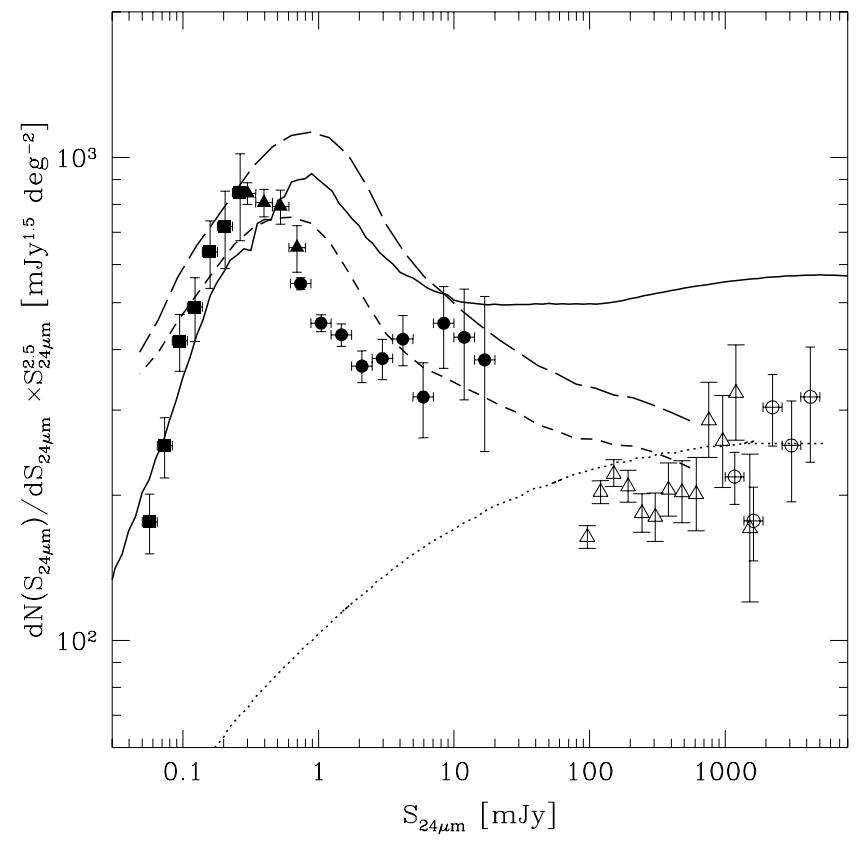

FIG. 3.-Differential extragalactic source counts at $24 \mu \mathrm{m}$ for the main survey (filled circles), the verification strip (filled triangles), and ELAIS-N1 (filled squares), normalized to the Euclidean distribution $\left[N(S) \propto S^{-2.5}\right]$. The IRAS data points (Hacking \& Soifer 1991, open triangles; Sanders et al. 2003, open circles) transformed to $24 \mu \mathrm{m}$ are shown. The counts are compared to the predictions of LDP03 (solid line), Franceschini et al. (2001, long-dashed line) and Rodighiero et al. (2004, short-dashed line). The no-evolution model normalized to the IRAS counts is shown as a dotted line.

and $0.9 \mathrm{mJy}$, all counts can be fitted by super-Euclidean power laws:

$$
\frac{d N}{d S}= \begin{cases}(724 \pm 97) S^{-2.3 \pm 0.6} & 0.1<S<0.2 \\ (416 \pm 3) S^{-2.9 \pm 0.1} & 0.2<S<0.9\end{cases}
$$

The differential counts, normalized to the expected differential counts in a Euclidean universe, are shown in Figures 3 and 4 . At faint fluxes $(\sim 0.1 \mathrm{mJy})$, the average closest neighbor separation is $\sim 17^{\prime \prime}$, i.e., 0.12 sources per beam. This value is less than the estimated number of sources per beam at the $5 \sigma$ confusion level (assuming a slope of 1.5 below $0.1 \mathrm{mJy}$; Takeuchi \& Ishii 2004).

The predictions from the infrared galaxy evolution models of Franceschini et al. (2001, hereafter F01), Rodighiero et al. (2004), and LDP03 are displayed in Figure 3. The LDP03 model considers two populations of galaxies: nonevolving normal spirals and starburst galaxies whose luminosity density evolves with redshift. The F01 model uses three populations evolving differently: nonevolving normal spirals, a fast evolving population which includes type II active galactic nuclei (AGNs) and starburst galaxies, and type I AGNs. The type I AGNs evolution is based on the results from optical and X-ray observations. The F01 model has recently been updated (Rodighiero et al. 2004) with a different normalization to take into account the corrected counts from the reanalysis of the ELAIS-S1 (Gruppioni et al. 2002) and the Lockman Hole (Rodighiero et al. 2004) fields. In addition to the counts, these models fit the redshift distributions and the cosmic infrared background spectrum.

The deep ISOCAM $15 \mu \mathrm{m}$ counts (Elbaz et al. 1999; Rodighiero et al. 2004; Gruppioni et al. 2002; Metcalfe et al. 2003), transformed to $24 \mu \mathrm{m}$, are overplotted on the Spitzer 


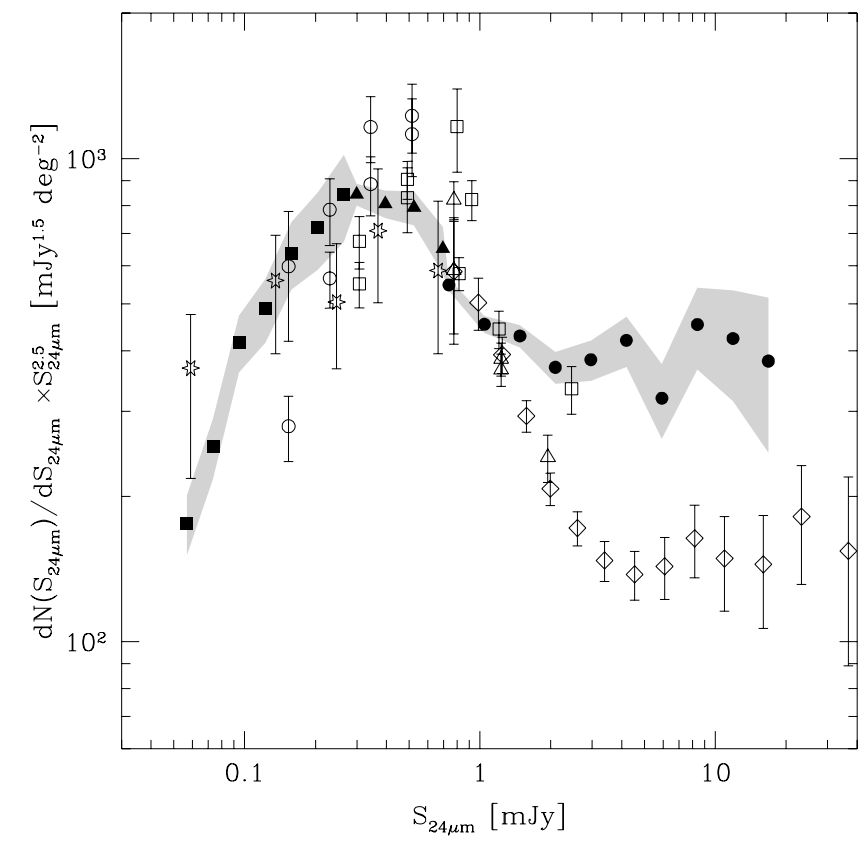

FIG. 4.-Spitzer FLS counts (same symbols as in Fig. 3, shaded region defines the error bars) are compared to the ISOCAM counts transformed to $24 \mu \mathrm{m}$ in the Hubble Deep Field-North and Hubble Deep Field-South (open circles; Elbaz et al. 1999), Marano Deep and Ultra Deep fields (open squares; Elbaz et al. 1999), in the Lockman Hole (open triangles; Rodighiero et al. 2004), in ELAIS-S1 (open diamonds; Gruppioni et al. 2002), and through the clusters A2390, A2218, and A370 (open stars; Metcalfe et al. 2003).

FLS $24 \mu \mathrm{m}$ counts in Figure 4 . The 15-24 $\mu \mathrm{m}$ flux transformation was done using template spectral energy distributions (SEDs) of three infrared luminous galaxies with $L_{\mathrm{IR}}=$ $10^{12}$ (ULIRG), $10^{11}$ (LIRG), and $10^{10}$ (starburst) $L_{\odot}$, taken from Chary \& Elbaz (2001). The $24 / 15 \mu \mathrm{m}$ flux ratio remains fairly constant in the redshift region $0.1-1.2$ except around $z=0.5$, where the PAH emission bands at $\sim 15 \mu \mathrm{m}$ enter the $24 \mu \mathrm{m}$ filter while the silicate absorption feature enters the $15 \mu \mathrm{m}$ filter. The median value we used to transform the ISOCAM counts is 1.2. The IRAS $25 \mu \mathrm{m}$ counts, appearing in Figure 3, are taken from Hacking \& Soifer (1991) and, for the brightest fluxes, are based on the revised IRAS bright galaxy sample of Sanders et al. (2003). These counts were transformed to $24 \mu \mathrm{m}$ counts using the same set of template SEDs $(25 / 24 \mu \mathrm{m}$ flux ratio of 1.8$)$.

The $24 \mu \mathrm{m}$ counts confirm the existence of the rapidly evolving dust-obscured population discovered by ISOCAM (Elbaz et al. 1999). The main difference between the model predictions and the data, which is reflected also in the direct comparison with the transformed ISOCAM counts, is a shift in the turnaround of the Euclidean-normalized counts. The peak in the $24 \mu \mathrm{m}$ counts (at $\sim 0.2 \mathrm{mJy}$ ) is fainter than that predicted by the models. It is highly improbable that this difference is due to problems in flux measurement and/or calibration. The $24 \mu \mathrm{m}$ calibration is accurate at the level of a few percent, while the uncertainty associated with flux measurements is less than $10 \%$ (see $\S 3$ ). The possibility of a systematic underevaluation of fluxes of slightly extended sources (which we extract as point sources with StarFinder) is also discarded by the good agreement with aperture measurements of faint sources.

It is possible that the two bands are sampling the same population of dust-obscured galaxies at $z \sim 1$, but the $15 \mu \mathrm{m}$ band is missing many objects around $z=1$ and all the galaxies at $z>1.5$. In fact, only $\sim 60 \%$ of the cosmic infrared background at $15 \mu \mathrm{m}$ has been resolved by the ISO observations (Elbaz et al. 2002). This hypothesis is consistent with the shift observed in the peak of the $24 \mu \mathrm{m}$ counts to fainter fluxes compared to the peak observed in the $15 \mu \mathrm{m}$ counts. Crucial information about the redshift distribution of the $24 \mu \mathrm{m}$ sources will come from deep spectroscopic observations and photometric redshift studies.

We thank A. Noriega-Crespo for useful discussions and the referee M. Malkan for insightful comments. We are grateful to H. Dole and A. Franceschini for providing us with their model predictions. This work is based on observations made with the Spitzer Space Telescope, which is operated by the Jet Propulsion Laboratory, California Institute of Technology, under NASA contract 1407. Support for this work was provided by NASA through an award issued by JPL/Caltech.
Aloisi, A., et al. 2001, AJ, 121, 1425

Bertin, E., \& Arnout, S. 1996, A\&AS, 117, 393

Calzetti, D. 1997, AJ, 113, 162

Chapman, S., Helou, G., Lewis, G., \& Dale, D. 2003, ApJ, 588, 186

Chary, R., \& Elbaz, D. 2001, ApJ, 556, 562

Condon, J. J., Cotton, W. D., Yin, Q. F., Shupe, D. L., Storrie-Lombardi, L. J., Helou, G., Soifer, B. T., \& Werner, M. W. 2003, AJ, 125, 2411

Diolaiti, E., Bendinelli, O., Bonaccini, D., Close, L., Currie, D., \& Parmeggiani, G. 2000, A\&AS, 147, 335

Elbaz, D., Cesarsky, C. J., Chanial, P., Aussel, H., Francheschini, A., Fadda, D., \& Chary, R. R. 2002, A\&A, 384, 848

Elbaz, D., Cesarsky, C. J., Fadda, D., et al. 1999, A\&A, 351, L37

Fadda, D., Jannuzi, B., Ford, A., \& Storrie-Lombardi, L. J. 2004, AJ, 128, 1

Fazio, G., et al. 2004, ApJS, 154, 10

Flores, H., et al. 1999, ApJ, 517, 148

Franceschini, A., Aussel, H., Cesarsky, C. J., Elbaz, D., \& Fadda, D. 2001, A\&A, 378, 1

Gardner, J. P., Cowie, L. L., \& Wainscoat, R. J. 1993, ApJ, 415, L9

Gruppioni, C., Lari, C., Pozzi, F., Zamorani, G., Franceschini, A., Oliver, S., Rowan-Robinson, M., \& Serjeant, S. 2002, MNRAS, 335, 831

\section{REFERENCES}

Hacking, P. B., Houck, J. R., \& Condon, J. J. 1987, ApJ, 316, L15

Hacking, P. B., \& Soifer, B. T. 1991, ApJ, 367, L49

Lagache, G., Dole, H., \& Puget, J.-L. 2003, MNRAS, 338, 555

Lilly, S., Le Fèvre, O., Hammer, F., \& Crampton, D. 1996, ApJ, 460, L1

Metcalfe, N., Shanks, T., Fong, R., \& Roche, N. 1995, MNRAS, 273, 257

Metcalfe, L., et al. 2003, A\&A, 407, 791

Oliver, S., et al. 2000, MNRAS, 316, 749

Rieke, G., et al. 2004, ApJS, 154, 25

Rodighiero, G., et al. 2004, A\&A, submitted

Sanders, D., Mazarrella, J., Kim, Surace, J. A., \& Soifer, B. T. 2003, AJ, 126,1607

Soifer, B. T., Neugebauer, G., \& Houck, J. R. 1987, ARA\&A, 25, 187

Takeuchi, T. T., \& Ishii, T. T. 2004, ApJ, 604, 40

Werner, M., et al. 2004, ApJS, 154, 1

Xu, C. K., Lonsdale, C. J., Shupe, D. L., Franceschini, A., Martin, C., \& Schiminovich, D. 2003, ApJ, 587, 90

Yan, L., McCarthy, P. J., Storrie-Lombardi, L. J., \& Weymann, R. J. 1998, ApJ, 503, L19 